\title{
ON THE QUOTIENT OF ENTIRE FUNCTIONS OF LOWER ORDER LESS THAN ONE
}

\section{BY ANDERS HYLLENGREN}

Communicated by Maurice Heins, March 9, 1962

The origin of this note is a question in the theory of functions: "If $f_{1}(z)$ and $f_{2}(z)$ are two entire functions of lower order less than one and if $f_{1}(z)$ and $f_{2}(z)$ have the same zeros, is $f_{1}(z) / f_{2}(z)$ a constant?" This is one of 25 problems published in Bulletin of the American Mathematical Society, January 1962, pp. 21-24.

The solution of this problem is that the quotient $f_{1}(z) / f_{2}(z)$ is not necessarily a constant. It is even possible to find such entire functions of lower order zero. To do this we introduce some definitions.

$$
\begin{aligned}
a_{n}=2^{(4 n) !}, \quad b_{n} & =2^{(4 n+2) !}, \quad P_{n}(z)=\left(1-\frac{z}{a_{n}}\right)^{a_{n}}\left(1+\frac{z}{b_{n}}\right)^{b_{n}}, \\
f_{1}(z) & =\prod_{n=1}^{\infty} P_{n}(z), \quad f_{2}(z)=e^{-z} f_{1}(z) .
\end{aligned}
$$

Now $f_{1}(z)$ and $f_{2}(z)$ are different entire functions with the same zeros. We denote

$$
M_{v}(r)=\max _{|z|=r}\left|f_{v}(z)\right|, \quad v=1,2 .
$$

For $r=2^{(4 m+3) !}$ the rough estimate

$$
\frac{\log \log M_{1}(r)}{\log r}<\frac{1}{m}
$$

implies that $f_{1}(z)$ is of lower order zero.

For $r=2^{(4 m+1) !}$ we obtain

$$
\frac{\log \log M_{2}(r)}{\log r}<\frac{1}{m}
$$

which implies that $f_{2}(z)$ is of lower order zero.

Thus the problem is solved.

The Royal Institute of Technology, Stockholm 